\title{
Feasibility Study of Backtracking Algorithm for Virtual Cluster Migration in Cloud Computing
}

\author{
T.Lavanya Suja \\ M.Phil.Scholar, Mother Teresa Women's \\ University,Kodaikanal,Tamilnadu,India
}

\author{
V.Savithri \\ Research Supervisor, Women's Christian \\ College,Nungambakkam,Chennai,Tamilnadu,India
}

\begin{abstract}
Cloud Computing (CC) is an emerging field in which research scholars from academia and industry professional contribute their knowledge to understand and propagate the dimensions of its applications. One of the popular services in CC is Infrastructures as a Service (IaaS) by which the customers can rent a highly configured server, storageetc. from the providers. On the provider's side in order to maintain the numerous servers for myriad reasons like load balancing, faulttolerance, complying to the Service Level Agreements(SLA), they have to migrate these servers from one Physical Machine(PM) to another PM. A Backtracking algorithm is proposed and its feasibility study is done by comparing the approach and working with various similar existing algorithms.
\end{abstract}

\section{Keywords}

VC migration, virtualcluster, virtual machines, cloud computing, backtracking algorithm, VC, VM, PM

\section{INTRODUCTION}

Cloud Computing is defined in various ways and a comprehensive definition is given by [10] National Institute of Standards and Technology (NIST), USA as "Cloud computing is a model for enabling convenient, on-demand network access to a shared pool of configurable computing resources (e.g., networks, servers, storage, applications, and services) that can be rapidly provisioned and released with minimal management effort or service provider interaction. This cloud model promotes availability and is composed of five essential characteristics, three service models, and four deployment models.

Followingthis definition we come to know that $\mathrm{CC}$ has 3 service models namely

- $\quad$ Software as a Service(SaaS)

- Platform as a Service(PaaS)

- Infrastructure as a Service(IaaS)
In this paper the concern is in IaaS where the consumer rents servers of any configuration to suit their needs instead of buying upfront there by saving the capital expenditure after signing the SLA.As a result of it the maintenance also move to the responsibility of the provider hence the consumer is free off with only involved in the working and development of applications in the servers.

On the provider's perspective he has the huge share of work in building, maintaining and living up to the SLA all the time the servers are "on". A previous research paper [11] surveyed 30 research papers and compiled the information regarding the concepts, working and approach of the IaaS and the algorithms behind it. As a next step it is found that the Backtracking algorithm as a potential candidate for providing better solutions for migration of servers from one PM to another PM.

\subsection{Migration}

Migration is a process of moving a machine from one place to another logically. In Iaas there are umpteen numbers of servers of configuration sizes small, big and large with the providers which are called Virtual Machines (VM), As a matter of fact and convenience to suit the practical needs of the consumers often they are grouped as Virtual Clusters (VC) where a group of VM belongs to one cluster. One or more VCs usually reside in a PM. During the course of working these VCs need to be migrated from one PM to another PM for various reasons like

- Load Balancing

- Fault Tolerance

- Complying to the SLA

- Better Performance,etc

At this juncture the migration algorithms come into picture. The below figures(See figure-1\&figure-2) adds to the understanding of the explanation above. 

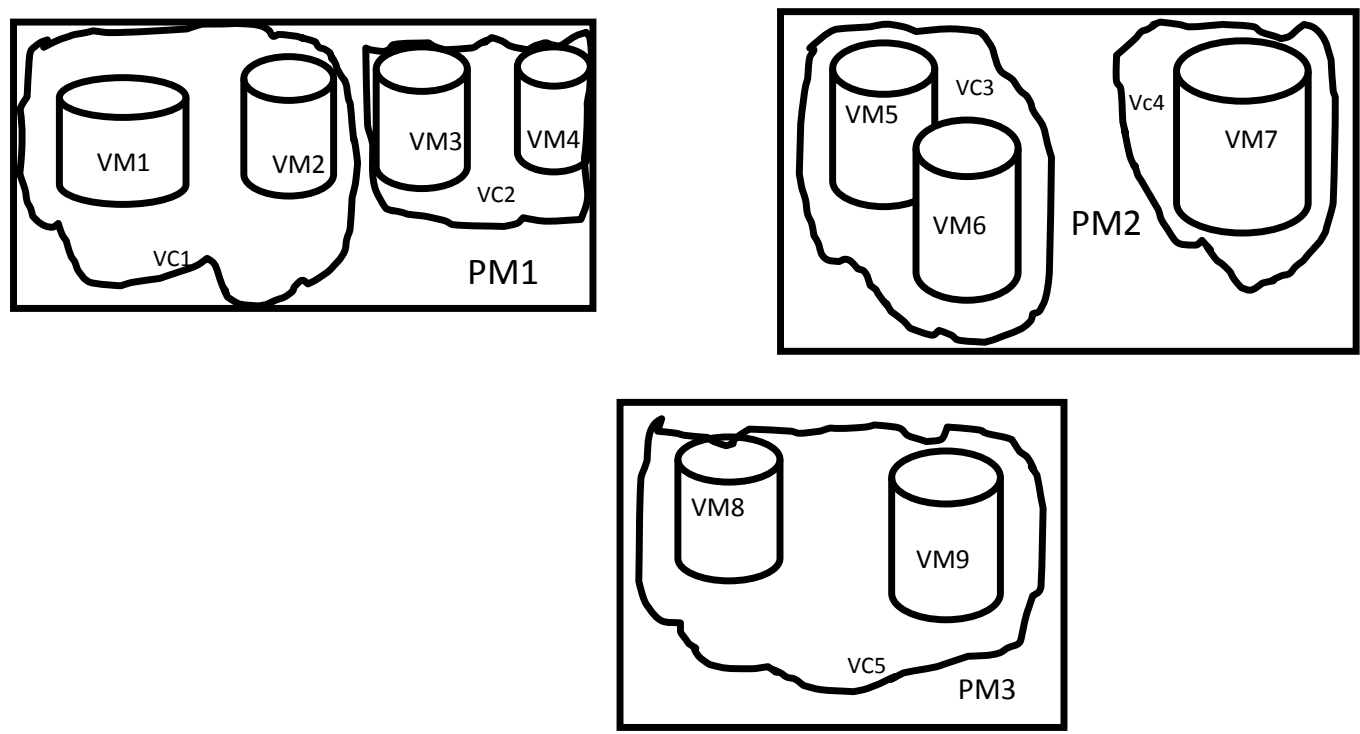

Fig 1: Before Migration
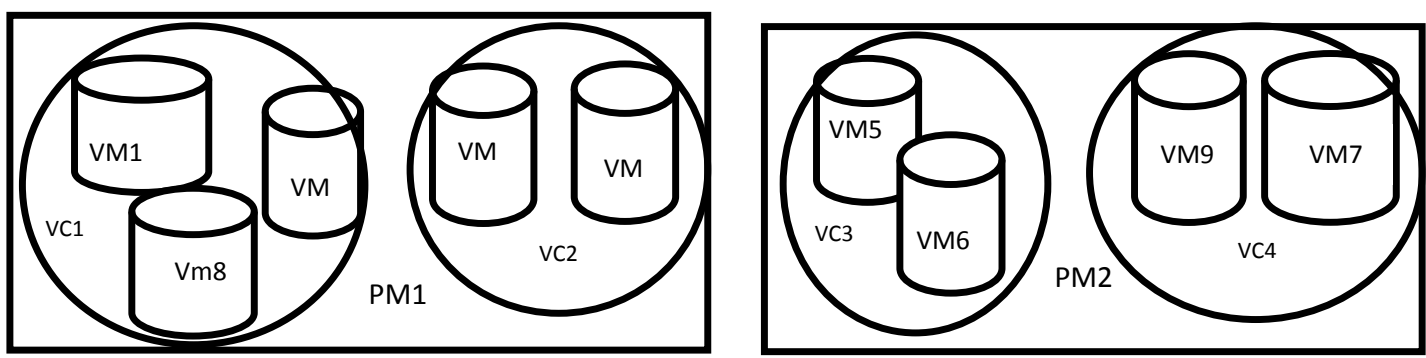

Fig2: After Migration due to Fault tolerance

As we can see from the above figures that after migration PM3's 2 VMs are distributed to PM1 and PM2 as for the reason due to Pm3's failure. As a result of it the applications and the users experience little downtime and was able to continue working despite their PM's failure. The same theory of migration can occur in the case of other chances like Load balancing and complying with SLA,etc.

\section{LITERATURE REVIEW}

In [1] the authors compare and contrast the SOA andCC by 4 factors such as drivers,provisioning,design principles and crosscutting concerns. There by they bring out the fact that $\mathrm{CC}$ belongs to the context of SOA. They introduce a new Cloud-RMM (Cloud Reference Migration Model) which can be used as a reference model to categorize the research in terms of different processes and crosscutting concerns. As a result they conclude by saying that $\mathrm{CC}$ was in a formative stage then so the necessary things are common research agenda and automation in cloud migration which help in the future development of CC.

In [2] a survey conducted by Susan Sutherland and Girija Chetty within the Australian Capital Territory, the authors reveal the fact that about $71 \%$ of the companies are ready to migrate to Cloud. They bring out the fact that there is a lack of standard based interoperability protocols and a progress gap in the research for convergence of cloud with other applications.

The performance analysis done in [3] statistically says that a 3 seconds of downtime was experienced in a 44 seconds long migration time ina Moving Virtual Cluster(VC) from one Physical Machine(PM) to other PM is Migration

Generic Algorithms and various approaches are surveyed for their working and performance. A new approach called Backtracking algorithm for VC migration is proposed

The Backtracking algorithm provides a better solution when compared with previous algorithms by taking into account the parameters such as memory size,downtime,performance etc.web2.0 cloud applications. The fact also proves that SLA of $90 \%$ is not violated at all while a stringent $99 \%$ of SLA is violated for a short moment. The data obtained from this analysis discloses that live migration is inevitable in VM migration.

In another paper Matthias et al. [4] propose many approaches for efficient distribution of VMs to compute nodes in a cloud computing environment. In their implementation they observed peer-to-peer distribution based on BitTorent method suits well for remote sites, multicast method shows better performance than others. The only disadvantage of their research is that they have to be tested yet. This research though reiterates the fact that VM distribution is an important factor in CC.

In a work very similar to that of ours the authors of [5] have come up with a Dynamic Threshold(DT) algorithm for VM consolidation to pave way for energy efficiency and green computing in CC.The experimentation results shows that the proposed algorithm outperforms the Non-Power Aware Policy(NPA) algorithms and also does better in terms of SLA violation levels. This research closely related to ours except the fact our algorithm is for VM migration. 
Bram Rongen in [6] carried out a case study for an organization called Bizzdesign to find out how useful was a Cloud Adoption Toolkit in migrating on-premises application to cloud. The 5 phases as specified in the toolkit are evaluated by conducting semi structured interviews with the CTO of the organization. The net result gave them a road map for migration though not very specific. This case study emphasize the fact that the crucial decision of migration to $\mathrm{CC}$ in an organization is a month long process and so after their decision it is the provider's duty to meet up the SLA as agreed upon.

Rajkumar et al discusses in another paper [7] that energy efficiency is a key component to be considered in migration of VMs but without performance trade off. They experimented the same with Online Optimal Deterministic Algorithm and added that the algorithm should be adaptive in nature so as to avoid the performance tradeoff, reduction in violation of SLAs and number of VM migrations. The simulation was done using CLOUDSIM. Though it is related to our research it differs from the fact that ours aim for VC migration which is a group of VMs and hence it is better than the former as it saves time.

Wenying Zeng et al. [8]analyses the underlying concepts and working of cloud computing systems and proposes a layered architecture of cloud storage. The operation discussed about

\begin{tabular}{|c|c|c|c|c|}
\hline AUTHORS & ALGORITHM & APPROACH & ADVANTAGE & DISADVANTAGE \\
\hline $\begin{array}{l}\text { Vicar Yadav, Pooja Malik and Ajay } \\
\text { Singh Chauhan [12] }\end{array}$ & Power Nap & $\begin{array}{l}\text { Ant Colony } \\
\text { Optimization } \\
\text { and Bee Hive } \\
\text { Optimization }\end{array}$ & Less Power & More Hardware \\
\hline $\begin{array}{l}\text { Ajeena Beegom and M S Rajasree } \\
\text { [13] }\end{array}$ & Greedy & Centralized & Less cost & $\begin{array}{l}\text { Probability of Load } \\
\text { Prediction Module's } \\
\text { failure }\end{array}$ \\
\hline $\begin{array}{l}\text { Anton Belaglosav and Rajkumar } \\
\text { Buyya[14] }\end{array}$ & Generic & Optimal & $\begin{array}{l}\text { Better } \\
\text { performance and } \\
\text { complying with } \\
\text { SLA }\end{array}$ & - \\
\hline AUTHORS & ALGORITHM & APPROACH & ADVANTAGE & DISADVANTAGE \\
\hline Parampreet Singh Jaura[15] & Generic & $\begin{array}{l}\text { Load Balancing } \\
\text { based on time } \\
\text { zones }\end{array}$ & $\begin{array}{l}\text { Easy } \\
\text { implementation }\end{array}$ & Change in locality \\
\hline $\begin{array}{l}\text { Somaeyh Soltan Bagshahi et } \\
\text { al.[16] }\end{array}$ & Greedy & Feasible & $\begin{array}{l}\text { Better } \\
\text { performance and } \\
\text { Time saving }\end{array}$ & $\begin{array}{l}\text { Only size of VM and } \\
\text { PM are considered }\end{array}$ \\
\hline
\end{tabular}

Ant Colony Optimization which was also quoted by authors of [12] in their research work as a better candidate for consideration. The fact that how data can be migrated carefully and wisely is discussed here.

SnowFlock is a variant of VM Fork developed by authors of [9] who advocates the fast that it takes less than a second (very less time) when compared with traditional VM Fork. Also they include the fact that it takes only memory in the order of fewer MBs in contrary to the previous Forks taking Few GBs. Multicasting is also available in SnowFlake Fork method which localizes the cloned VMs as to sophisticate for easy movement and saving time. The results show us that there are so many ways to replicate VMs when needed and also it is very essential in CC.

\section{COMPARISIONOF EXISTING ALGORITHMS}

In the below table a comparison analysis of few prominent and is compared with other equivalent approaches existing in the field. This analysis proves the fact that one approach is better than the other in some regard e.g., hardware, performance, time, energy, etc. algorithms is tabulated. Their working and approach is studied 


\begin{tabular}{||l|l|l|l|l||}
\hline Pallavi Gupta et al.[17] & Generic & $\begin{array}{l}\text { Green } \\
\text { Computing }\end{array}$ & $\begin{array}{l}\text { Less Heat and } \\
\text { Fewer PMs }\end{array}$ \\
\hline $\begin{array}{l}\text { Gulshan Soni, Mala Kalra, } \\
\text { Pradip et al. [18] } \\
\text { (Who Surveyed) }\end{array}$ & $\begin{array}{l}\text { VM Live } \\
\text { Migration }\end{array}$ & Pre-copy & Less downtime \\
Time overhead in re- \\
copying of Dirty pages
\end{tabular}

\section{FINDINGS}

There is always a need of new methods to drive the future of an emerging technology,so is true for migration in CC. After carefully understanding and comparing the various existing algorithms we would find rather a gap between performance and time. In our previous work [11] we came to know that Backtracking approach have not been tried so far and found it interesting to dig upon. So in this paper we propose the expanded code of Backtracking algorithm and found that working fine for our random data. Though Greedy approach [13] and [16] proposed is simple to implement and produces a feasible solution but it does not take into account of any of the SLA constraints except the size of VM and PM.

\section{BACKTRACKING ALGORITHM}

\section{Aim}

Migrate VCs from one PM to another PM based upon the constraints.

2. Explicit Constraints

Select VMs from the same VCs

3. Implicit Constraints

- $\quad$ Memory size of VM and PM

- Minimum down time

\section{Pseudocode}

- Increase in performance after migration

Procedure VC_Migration( )

Begin

vclist,pmlist arranged in descending order;

VMs arranged in ascending order of downtime;

For each PM in pmlist do

While sizeof $(\mathrm{PM})>\operatorname{minsizeof}(\mathrm{vclist}) \mathrm{do}$

For each VC in vclist do

If $(\operatorname{sizeof}(\mathrm{VC})>\operatorname{sizeof}(\mathrm{PM}))$ or $(\mathrm{flag}(\mathrm{vc})=$ 'done' )then continue; //backtrack

else

For each VM in VC do

$\operatorname{sizeof}(\mathrm{PM})=\operatorname{sizeof}(\mathrm{PM})-\operatorname{sizeof}(\mathrm{VM})$;

Endfor

Endif
Print "VC is in migration to PM"

Flag $[\mathrm{vc}]=$ "done";

Endfor

Endwhile

Endfor

End

\section{CONCLUSION AND FUTURE WORK}

The attempt to study the feasibility has come up with the coding and found satisfying enough to proceed further, Also the manual calculation of rough test data gave an optimal solution hence the feasibility study proved useful. The proposed Backtracking algorithm migrates one or more VCs from one PM to another PM after taking into account of criteria in SLA such as minimum downtime, memory size and VMs in same VC. In future it is aimed to implement this Backtracking algorithm with CLOUDSIM a simulating tool under Java platform and successfully come out with results. While working with the algorithm it is found that after migration few memory GBs are left unused which resembled the problem of internal fragmentation in Paging Memory. Similar solution like Compaction of Memory has to be thought for Consolidation of PMs in the future work.

\section{REFERENCES}

[1] Jamshidi.P,Ahmad.A,Pahl.C,CloudMigration Research: A Systematic Review (2014) ,IEEE Transactions on cloud computing Vol. 1 Issue: 2 ,pp. 142 - 157

[2] Susan Sutherland and Girija Chetty, Migration to Cloud Computing: a Sample Survey Based on a Research in Progress on the Investigation of Standard Based Interoperability Protocols for the Convergence of Cloud Computing, Service Oriented Architecture and Enterprise Architecture, International Journal of Information Processing \& Management; Feb2014, Vol. 5 Issue 1, pp.50-61.

[3] William Voorsluys, James Broberg, Srikumar Venugopal, Rajkumar Buyya ,Cost of virtual machine live migration in clouds: A performance evaluation(2009),Journal of Cloud Computing,pp. 254265,doi.10.1145/1890799/1890803

[4] MathiasSchmidt,NielsFallenback,MathewSmith,BerndFr eisleben, Efficient Distribution of Virtual Machines for Cloud Computing 2010 18th Euro micro International Conference on Parallel, Distributed and Network-Based $\begin{array}{lll}\text { Processing } & \text { (PDP),pp.567 } & \text { - }\end{array}$ doi.10.1109/PDP.2010.39 
[5] Anton Beloglazov, Rajkumar Buyya,Adaptive thresholdbased approach for energy-efficient consolidation of virtual machines in cloud data centers, JournalProceedings of the 8th International Workshop on Middleware for Grids, Clouds and e-Science,pp. 1-4

[6] Bram Rongen, Making the case for migration of Information systems to the cloud,(2012)16thTwente Student Conference on IT, Pages 6.

[7] AntonBeloglazov, RajkumarBuyya, Optimal online deterministic algorithms and adaptive heuristics for energy and performance efficient dynamic consolidation of virtual machines in Cloud data centers (2011),Concurrency and Computation: Practice and Experience,Vol. 24, Issue 13, pp. 1397-14207 ,DOI: $10.1002 /$ cpe. 1867

[8] Wenying Zeng, Yuelong Zhao, Kairi Ou, Wei Song, Research on cloud storage architecture and key technologies,(2009),Proceedings of $2^{\text {nd }}$ International conference on Interaction Sciences: InformationTechnology, culture and Human,pp.10441048.

[9] H. Andres Lagar-Cavilla, Joseph Whitney, Adin Scannell, Philip Patchin, Stephen M. Rumble, Eyal de Lara, Michael Brudno, M. Satyanarayanan ,Snow Flock: rapid virtual machine cloning for cloud computing",(2009), Proceedings of the 4th ACM European conference on Computer systems ,pp. 1-12 ,doi>10.1145/ pp.1519065.1519067

[10] http://csrc nist.gov/groups/SNS/cloud- computing/clouddef-v15.doc [online] Accessed:10.Mar.2015

[11] T.Lavanya Suja, V.Savithri," Analyzing a Novel Approach for VC migration in Cloud Computing", Proceedings of International Conference on Computing and Intelligent Systems(ICCIS 2015),International Journal of Computing Algorithm,Vol. 4 Special issue,pp. $1252-1256$

[12] Vikram Yadav, Pooja Malik, Ajay Singh Chauhan, Energy efficient VM optimization, International Journal of Computer Applications,(2014) Vol. 106 No.7, pp. $23-28$

[13] AjeenaBeegom, MS Rajasree,Resource provisioning and management for IaaS providers in cloud computing, (2014) International Journal of Computer Applications Vol. 104 No.17, pp.1-4.

[14] Anton Belaglosav, Rajkumar Buyya, Energy efficient resource management in virtualized cloud data centers, $10^{\text {th }}$ IEEE/ ACM International Conference on Cluster,Grid and Cloud Computing,2010,pp.826-831.

[15] Parampreet Singh Jaura,Time and load based cloud Scheduling algorithm,International Journal of Computer Applications (0975 8887) Vol. 110 No. 11, January 2015,pp.34-40

[16] Somayeh Soltan Baghshahi,Sam Jabbehdari,Sahar dabi,VM migration based on greedy algorithm in cloud computing,International Journal of Computer Applications (0975 8887) Vol. 96 No.12, June 2014,pp.32-35.

[17] PallaviGupta,Lokendra Vishwakarma,Awadheshwari Patel, Power aware VM consolidation considering multiple recourses with live migration. International Journal of Computer Applications ,Vol. 103 No 17, October 2014,pp.24-30.

[18] Gulshan Soni, Mala Kalra, Comparative study of live VM migration techniques in cloud. International Journal of Computer Applications, Vol. 84,No 14, December 2013,pp.19-25. 